\title{
Implementing adolescent SBIRT in an urban federally qualified health center: generalist vs. specialist service delivery models
}

\author{
Shannon Gwin Mitchell ${ }^{*}$, Arethusa S Kirk², Marla Oros ${ }^{3}$, Jan Gryczynski ${ }^{4}$, Kristi Dusek ${ }^{5}$, Colleen Hosler ${ }^{6}$, \\ Robert P Schwartz ${ }^{7}$, Barry S Brown ${ }^{8}$, Carolina Barbosa ${ }^{9}$, Laura J Dunlap ${ }^{10}$, David W Lounsbury ${ }^{11}$, Kevin E O'Grady ${ }^{12}$
}

From INEBRIA 12th Congress,

Atlanta, GA, USA. 24-25 September 2015

\section{Background}

Little is known about how best to implement SBIRT services in pediatric health care settings or who, optimally, should provide brief interventions when on-site behavioral health is available. The objective of this presentation is to present results from a cluster randomized trial examining implementation of adolescent SBIRT services for substance use within a US federally qualified healthcare system. Two different implementation models for conducting brief interventions (BIs) were compared using randomization at the clinic level to either: the Generalist Model (BI provided by primary care provider) or the Specialist Model (BI provided by behavioral health specialist).

\section{Material and methods}

Multilevel logistic regression modeling was used to examine differences by Condition in rates of successful delivery and documentation of the following services: (a) screening (of all adolescent patients ages 12-17), (b) brief advice (for patients reporting alcohol or drug use but scoring $\geq 2$ on the CRAFFT), and (c) brief intervention (patients scoring $<2$ on CRAFFT, delivered using either the Specialist or Generalist models). Due to the organization transitioning to a new electronic medical record (EMR) in month 6 of the study, data on BA and BI are currently limited to extractions from the new EMR.

\section{Results}

Multilevel logistic regression analyses taking into account the cluster-randomized design showed no significant differences between Generalist and Specialist

\footnotetext{
* Correspondence: smitchell@friendsresearch.org

${ }^{1}$ Friends Research Institute, Baltimore, USA

Full list of author information is available at the end of the article
}

conditions in rates of screening $(\mathrm{OR}=1.27 ; \mathrm{p}=.55)$, with significant volatility over time $(<.001)$ and variation by sites. In the post-EMR transition, Generalist sites were not significantly more likely to deliver appropriate BA $(\mathrm{OR}=1.34 ; \mathrm{p}=.70)$ or $\mathrm{BI}(\mathrm{OR}=1.53 ; \mathrm{p}=.36)$ than Specialist sites. Site-level intraclass correlations were higher than anticipated. Future analyses will examine practices for the full implementation period and subsequent to the removal of implementation support resources.

\section{Conclusions}

Both service delivery models showed promise for delivering BIs but the high rates of variability within sites demonstrate a need for further examination.

\section{Acknowledgements}

We thank Ms. Faye Royale-Larkins and the staff of Total Health Care for their collaboration on this implementation project. We also thank Drs. Tisha Wiley and Lori Ducharme for their continued guidance. The study was supported through National Institute on Drug Abuse (NIDA) Grant1R01DA034258-01 (PI Mitchell).

\section{Authors' details \\ ${ }^{1}$ Friends Research Institute, Baltimore, USA. ${ }^{2}$ Total Health Care, Baltimore, USA. ${ }^{3}$ Mosaic Group, Baltimore, USA. ${ }^{4}$ Friends Research Institute, Baltimore, USA. ${ }^{5}$ Friends Research Institute, Baltimore, USA. ${ }^{6}$ Mosaic Group, Baltimore, USA. ${ }^{7}$ Friends Research Institute, Baltimore, USA. ${ }^{8}$ University of North Carolina at Wilmington, Wilmington, USA. ${ }^{9}$ RTI International, Chicago, USA. ${ }^{10} \mathrm{RTI}$ International, Research Triangle Park, USA. ${ }^{11}$ Yeshiva University, Bronx, USA. ${ }^{12}$ University of Maryland, College Park, USA.}

Published: 24 September 2015

doi:10.1186/1940-0640-10-S2-O24

Cite this article as: Mitchell et al:: Implementing adolescent SBIRT in an urban federally qualified health center: generalist vs. specialist service delivery models. Addiction Science \& Clinical Practice 2015 10(Suppl 2):O24. 